\title{
FTIR Image Analysis and Visualization: A Multivariate Approach
}

\author{
William J. McCarthy, N. Simon Nunn, Nishikida Koichi, Eric Y. Jiang \\ Thermo Electron Corporation \\ 5227 Verona Road, Madison Wisconsin 53711
}

As the utility of infrared imaging has increased, samples that may not be ideal for spectroscopy, but are important for scientific or industrial reasons are being examined with more frequency. These samples often have morphological differences due to thickness, refractive index variation, and scattering that create artifacts, correlated noise and systematic errors. These effects can obscure chemical heterogeneity in the spectroscopic multidimensional imaging data. Although well-designed optical and electronic components can minimize these effects, they cannot be entirely eliminated. Consequently, distinguishing structure morphology from information about molecular specificity remains a challenge for many imaging samples. A polypropylene film sample showing imperfections was studied on an Imaging FT-IR Microscope system to find causes of imperfections.

Spectral processing techniques such as baseline correction, derivatives and smoothing have been successfully employed to enhance images and reveal important information about spatial distribution. Examples using these methods will be shown. Exploratory data analysis techniques (often designated as unsupervised learning), such as principal component analysis (PCA) and factor analysis, have the ability to extract information from multidimensional data. Reducing the dimensionality of the spectra by these techniques allows visualization of the data that is directly related to the problem to be solved. Examples using classification methods such as distance mapping to show correlation and reveal spatial structure and heterogeneity of chemical species in a sample will also be shown and discussed.

Infrared imaging can also be enhanced by using visual images and fluorescence imaging.

Although many imperfections looks similar by visible and even FT-IR images, some of them fluoresce by the illumination with UV light, while the other do not. Also, the colors of fluorescence may be different from place to place, showing different kind of information on the environment of contaminant, "fish-eyes: and gels. Images of distributions of contaminants such as polyamide, calcium carbonate, cellulose, and others were obtained.

The most important information obtained from the fluorescence image is that some portions of intruders are found to fluoresce, while the other portion of the same intruder does not fluoresce. Analyses of several intruders and their fluorescence environments provided a clearer picture telling what went wrong during the film manufacturing. 


\section{References}

(1) Douglas B. Murphy (2001), Fundamentals of Light Microscopy and Electronic Imaging, John Wiley and Sons, New York.

(2) Paul Geladi and Hans Grahn (1996), Multivariate Image Analysis, John Wiley and Sons, Chicester.

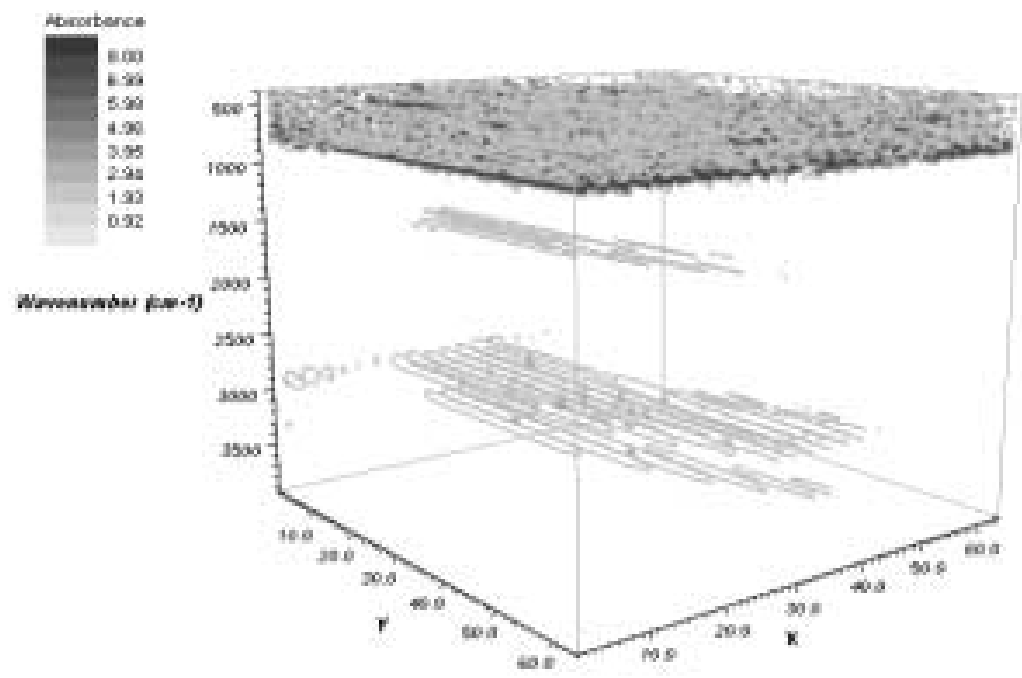

FIGURE 1. Three dimensions representation of a hypercube with $\mathrm{z}$-axis wavenumbers, $\mathrm{x}$ and y axes spatial dimension and absorbance plotted as false color. Selective wavenumbers (or planes) are removed to improve visibility.

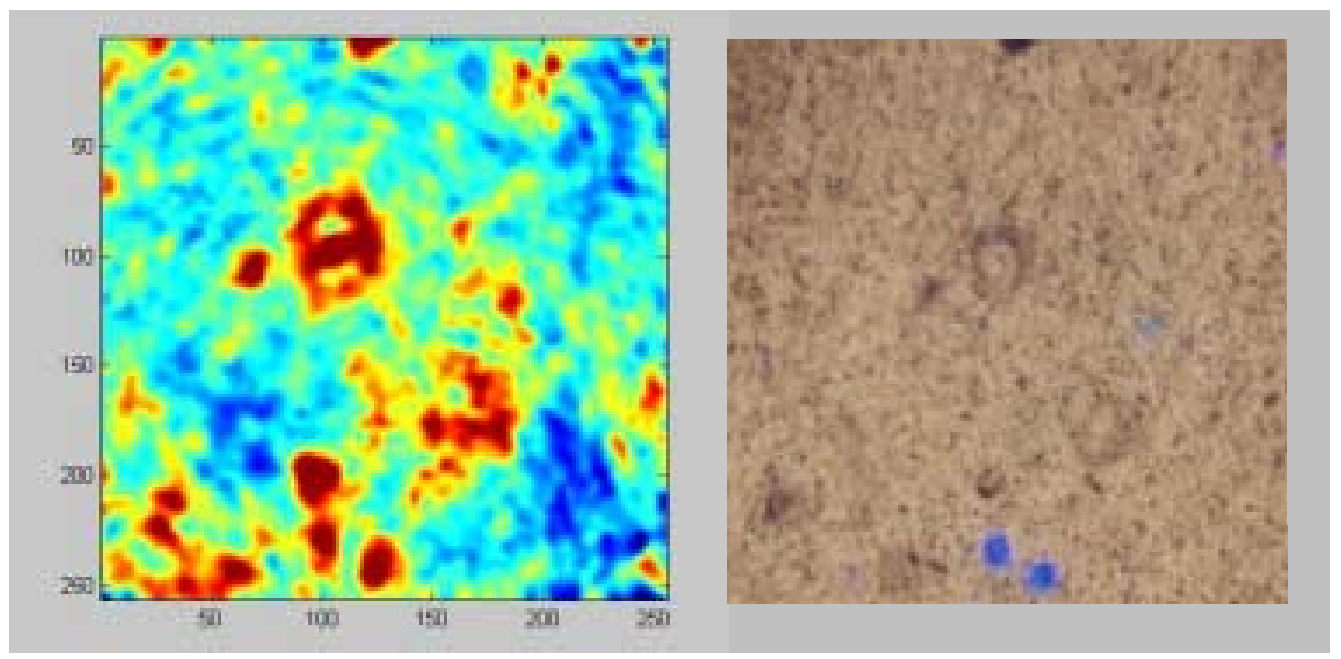

FIGURE 2. Absorbance image corresponds with the sum of visible and fluorescence image. 